\title{
Clinical observation of CT-guided intra-art
conventional radiofrequency and pulsed radiofrequency in the treatment of chronic sacroiliac joint pain
}

This article was published in the following Dove Press journal: Journal of Pain Research

\section{Yuanyuan Ding' \\ Hongxi Li' \\ Peng Yao' \\ Tao Hong' \\ Rongjie Zhao ${ }^{2}$ \\ Guangyi Zhao ${ }^{3}$}

'Department of Pain Management, Shengjing Hospital of China Medical University, Shenyang, China; ${ }^{2} \mathrm{Class}$ 5 of 2020 Session, Shenyang No. 20 High School, Shenyang, China; ${ }^{3}$ Department of Anesthesiology, Shengjing Hospital of China Medical University, Shenyang, China
Correspondence: Guangyi Zhao Department of Anesthesiology, Shengjing Hospital of China Medical University, No. 36, San Hao Street, Shenyang, Liaoning, II 0004, China

Email zhaoguangyil 10004@163.com

\begin{abstract}
Background: Sacroiliac joint pain (SIJP) is an important cause of low back pain and seriously affects the patients' quality of life. Therefore, it is urgent to find effective treatment methods. Objective: To observe the efficacy of intra-articular (IA) conventional radiofrequency (CRF) and pulsed radiofrequency (PRF) treatment of Sacroiliac joint syndrome (SIJS) under computed tomography (CT) guidance.
\end{abstract}

Study design: Retrospective comparative study.

Setting: Shengjing Hospital of China Medical University.

Patients and methods: Sixty-four patients with SIJS were enrolled in the Pain management. Patients were randomized into two groups: $\mathrm{CRF}$ (CRF group, $\mathrm{n}=32$ ) and PRF (PRF group, $\mathrm{n}=32$ ). At each observation time, the general condition, visual analog scale (VAS), the total efficiency rate, Oswestry disability index (ODI), and 36-item short-form health survey were followed up. Results: Compared to the pretreatment value, the VAS and the ODI decreased in both groups after treatment $(P<0.05)$. In the CRF group, the VAS and the ODI decreased significantly at 1 week after treatment $(P<0.05)$; at 6 and 12 months after treatment, the VAS and the ODI were lower than that in the PRF group $(P<0.05)$. The total efficiency rate in the CRF group and PRF group was $56.3 \%$ and $31.3 \%$, respectively $(P<0.05)$. After the relief of pain, both groups received different degrees of improvement in the quality of life. Compared to the pretreatment value, physical component summary (PCS) and the mental component summary (MCS) in both groups were increased after treatment $(P<0.05)$; in the CRF group, PCS and MCS increased significantly at 1 week after treatment $(P<0.05)$; and at 6 and 12 months after treatment, PCS and MCS were higher than those in the PRF group $(P<0.05)$.

Conclusion: $\mathrm{CT}$-guided IA PRF and CRF in the treatment of sacroiliac pain are safe and effective. CRF is superior to PRF in the early and late stage. It is recommended for the treatment of SIJP. Keywords: sacroiliac joint pain, conventional radiofrequency, pulsed radiofrequency, intraarticular, sacroiliac joint syndrome

\section{Introduction}

Sacroiliac joint pain (SIJP) is an important cause of low back pain. Acute or chronic injury to sacroiliac joint (SIJ) cartilage, joint capsule, peripheral ligament, and soft tissue occurs in SIJP followed by pain in the lumbosacral and lower extremities. ${ }^{1,2} \mathrm{At}$ present, $15 \%-30 \%$ of low back pain is caused by SIJ disease. ${ }^{3}$ This seriously affects the patients' quality of life and is also the main cause of early incapacity. There are many diseases causing SIJS, the pathogenesis is unclear, and the diagnosis is difficult. ${ }^{4}$ 
The course of SIJS tends to be long and difficult to cure. Therefore, it is urgent to find effective treatment methods.

SIJ block is a traditional treatment method. It can effectively relieve the SIJP, but the maintenance time is short, the long-term treatment effect is limited, and repeated treatment is needed. ${ }^{5}$ Radiofrequency treatment can alleviate neuropathic pain, knee pain, and so on ${ }^{6,7}$ and has gradually become a common technique for treating chronic pain. Radiofrequency includes conventional radiofrequency (CRF) and pulsed radiofrequency (PRF). Radiofrequency is minimally invasive. Testing before treatment can be accurately positioned, with the advantages of safety and repeatability. Therefore, it is widely used. The temperature range of $\mathrm{CRF}$ is $75-95^{\circ} \mathrm{C}$. The higher the temperature, the more serious damage to the nerve, and the higher the complications such as numbness. ${ }^{8}$ This also limits the scope of its application. The temperature of the PRF does not exceed $42^{\circ} \mathrm{C}$ and there is almost no damage to the nerve. PRF analgesic effect is independent of temperature but is related to neuromodulation. However, the effect is slow and the recurrence rate is high. ${ }^{9,10}$

There have been many reports on the treatment of SIJS by radiofrequency denervation (RFD), but RFD requires precise and careful selection of nerves. Intra-articular radiofrequency (IARF) treatment is safer than RFD, but there are few studies on IARF in the treatment of SIJP. This study was to observe the efficacy of intra-articular (IA) CRF and PRF treatment of
SIJS under computed tomography (CT) guidance. Oswestry disability index (ODI), the total efficiency rate, and qualityof-life improvement (36-item short-form health survey [SF-36]) were compared and observed for clinical outcome.

\section{Methods}

\section{Patients}

From January 2015 to December 2016, 64 patients with SIJS were enrolled in the Pain management, Shengjing Hospital of China Medical University. Patients were randomized into two groups according to the order of entry: CRF (CRF group, $n=32$ ) and PRF (PRF group, $n=32$ ) (Figure 1). The study was approved by the Ethics Committee of Shengjing Hospital of China Medical University. All patients were informed of risks and complications before treatment, and the written informed consent was obtained from all patients.

Inclusion criteria: conforming to IASP's definition of SIJP. ${ }^{11} 1$ ) the course of disease was more than 1 month; 2) pain in one or bilateral lumbosacral regions, severe patients with pain in the hips, groin, and lower extremities; 3) moderate to severe pain, the visual analog scale (VAS) of 24 hours average pain intensity scores was $>5$ points before enrollment; 4) physical examination: tenderness and percussion pain in the sacroiliac region, a positive result in at least one of the following tests (Patrick sign, compression and distraction test, Gaenslen sign), and no abnormalities in neurologic examination; 5) CT or magnetic resonance examination suggested

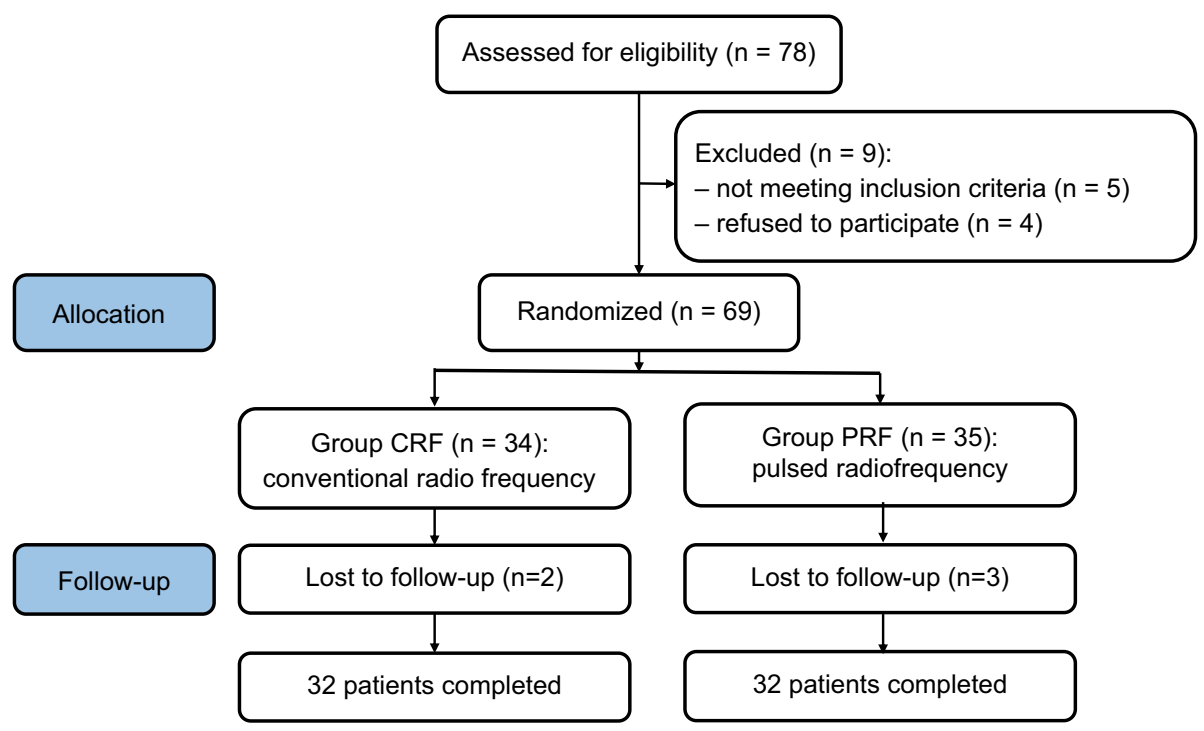

Figure I Schematic illustration of the study design.

Note: All 64 patients were included in the treatment.

Abbreviations: CRF, conventional radiofrequency; PRF, pulsed radiofrequency. 
sacroiliac arthritis; 6) IA block could relieve more than 50\% pain; and 7) conservative treatment was ineffective.

Exclusion criteria: 1) tumor, tuberculosis, postoperative SIJ pain, and other secondary SIJ lesions; 2) local puncture area infection; 3) patients with mental illness, mental retardation, and disturbance of consciousness; 4) severe liver, kidney, and cardiopulmonary diseases; 5) patients with abnormal coagulation function; and 6) patients with local anesthetic drug allergy.

\section{Treatment method}

The venous access was established, and ceftriaxone sodium was given to prevent infection at 30 minutes before treatment.

Under CT guidance, the patient was placed in a prone position and vital signs were monitored during the treatment. After local disinfection, $0.5 \%$ lidocaine was used for layerby-layer infiltration anesthesia. The radiofrequency needle was taken along the established puncture angle and path to the IA SIJ. CT scan was confirmed again to further adjust the needle directly to the IA SIJ. The RF needle was further connected to the RF instrument for testing: $50 \mathrm{~Hz}, 0.1-0.3$ $\mathrm{V}$ test sensation and $2 \mathrm{~Hz}, 0.1 \mathrm{~V}$ test exercise, no induction of hips and lower extremity muscle tremors and pain. The treatment was started after the position was satisfactory. 1) CRF group: started at $50^{\circ} \mathrm{C}$ and gradually heated up to the target temperature of $80^{\circ} \mathrm{C}$ for 180 seconds. 2) PRF group: $42^{\circ} \mathrm{C}$ PRF 600 seconds. After CRF or PRF treatment, both groups were injected with an analgesic complex solution (2\% lidocaine $1.5 \mathrm{~mL}+$ compound betamethasone $5 \mathrm{mg}+$ normal saline $0.5 \mathrm{~mL}) 3 \mathrm{~mL}$. The needle was removed and the puncture point was pressed. The patient was observed for 15 minutes and then returned to the ward.

\section{Observations and follow-up}

General condition was recorded before treatment, including age, gender, pain duration, VAS, pain position, and pain side. Follow-up evaluations were performed at 1 week, 2 weeks, 1 month, 3 months, 6 months, and 12 months, respectively. Evaluations were followed up by medical staffs of the nonsurgical group using a double-blind approach.

1. VAS pain score: 0 points (painless) to 10 points (intolerable pain).

2. The total efficiency rate: according to the WHO assessment criteria for pain relief, the efficacy was divided into four levels. Subjective symptoms and clinical signs were assessed at 12 months, divided into complete remission (CR), partial remission (PR), mild remission (MR), and no remission (NR). CR: pain disappeared; PR: pain was significantly relieved; MR: pain was relieved; and NR: pain was not relieved, and sometimes autonomic dysfunction suffered. The total efficiency rate $(\%)=[(\mathrm{CR}+\mathrm{PR}+$ MR) $/ \mathrm{n}] \times 100 \%$.

3. ODI: assessed the disability and pain levels. ODI consisted of two questions, including pain intensity, self-care, lifting, walking, sitting, standing, interfering with sleep, sexual life, social life, and tourism. Each question had six options and was scored as $0-5$ options. The sum of scores was expressed as a percentage: $0 \%$ represented no pain or disability, while $100 \%$ represented the most severe pain and disability.

4. SF-36 assessment ${ }^{12}$ : assessed the quality of life before and after treatment, including physical and mental status. The physical state included physical function, physical role, bodily pain, and general health; mental state included vitality, social function, emotional role, and mental health. Finally, the physical component summary (PCS) and the mental component summary (MCS) were calculated.

\section{Statistics}

SPSS18.0 statistical software (IBM Corporation, Armonk, NY, USA) was used to analyze the data. The single-sample Kolmogorov-Smirnov test was used to test the normality of the measurement data. The normal distribution variables were compared using one-way ANOVA followed by least significant difference pairwise comparison; values were expressed as the mean $\pm \mathrm{SD}(\bar{x} \pm \mathrm{SD})$. The abnormal distribution variables were compared using the Kruskal-Wallis rank sum test; values were expressed as the median $\pm I Q R$. Chi-square test or Fisher's exact test was used to analyze the enumeration data. $P<0.05$ was statistically significant.

\section{Results}

\section{General condition before treatment}

There was no significant difference in the general condition between two groups before treatment, including age, gender, pain duration, VAS, and pain position and side $(P>0.05)$ (Table 1).

\section{Intratreatment patient condition}

All patients completed the treatment. Under the guidance of $\mathrm{CT}$, the needle was inserted at a specific angle and depth. The CT scan showed that the tip of the needle was located in the IA SIJ. The three-dimensional CT could further clarify the position, showing that the RF needle was located at the SIJ. Tremors and painful sensations in the hips and lower extremity muscles were not induced by the sensory and exercise 
tests. Patients in both groups had no serious complications such as spinal cord injury and limb weakness after treatment (Figure 2).

\section{VAS before and after treatment}

At each observation point, the VAS in both groups decreased after treatment. Compared to pretreatment value, the difference was significant $(P<0.05)$. CRF group had a rapid onset, and VAS decreased significantly at 1 week after treatment. Compared to the PRF group, the difference was statistically significant $(P<0.05)$; the PRF group had a slow onset, and VAS decreased gradually. At 2 weeks, and 1 and 3 months, there was no significant difference between the two groups $(P>0.05)$. The pain relief in the CRF group was maintained for a long time. At 6 and 12 months after treatment, the VAS was lower than that in the PRF group, and the difference was significant $(P<0.05)$ (Figure 3$)$.

\section{The total efficiency rate}

The total efficiency rate in the CRF group and PRF group was $56.3 \%$ and $31.3 \%$, respectively. The difference between the two groups was significant $(P<0.05)$ (Table 2$)$.

\section{ODI before and after treatment}

At each observation point, the quality of life in both groups improved and the ODI decreased after treatment. Compared to the pretreatment value, the difference was significant $(P<0.05)$. The CRF group improved quickly, and ODI decreased significantly at 1 week after treatment. Compared to the PRF group, the difference was significant $(P<0.05)$.

Table I General condition before treatment

\begin{tabular}{|l|l|l|}
\hline Parameters & Group & PRF \\
\cline { 2 - 4 } & CRF & 32 \\
Patients (n) & 32 & $11(34.4)$ \\
Gender (n, \%) & $13(40.6)$ & $21(65.6)$ \\
$\quad$ Female & $19(59.4)$ & $50.45 \pm 5.45(43-57)$ \\
Male & $49.68 \pm 5.23(42-56)$ & $15.92 \pm 3.53(11-20)$ \\
Age (years, range) & $15.67 \pm 3.19(10-21)$ & $7.12 \pm 1.17$ \\
Pain duration before treatment (months, range) & $7.05 \pm 1.25$ & \\
VAS before treatment & & $9(28.1)$ \\
Side (n, \%) & $11(34.4)$ & $12(37.5)$ \\
$\quad$ Right & $9(28.1)$ & $11(34.4)$ \\
$\quad$ Left & $12(37.5)$ & \\
Both & & $32(100)$ \\
Pain position & $32(100)$ & $8(25.0)$ \\
$\quad$ Hips & $10(31.3)$ & $8(25.0)$ \\
$\quad$ Groin & $7(21.9)$ & \\
Lower extremities & & \\
\hline
\end{tabular}

Note: Data are presented as numbers ( $n$, \%) of patients or mean \pm SD.

Abbreviations: CRF, conventional radiofrequency; PRF, pulsed radiofrequency; VAS, visual analog scale.


Figure 2 (A) CT scan showed that the tip of the needle was located in the intra-articular sacroiliac joint, as indicated by the white arrows; (B) three-dimensional CT reconstruction showed that the radiofrequency needle was located at the sacroiliac joint, as indicated by the black arrows.

Abbreviation: CT, computed tomography. 
The quality of life in the PRF group improved slowly and the ODI decreased gradually. At 2 weeks, and 1 and 3 months, there was no significant difference between the two groups $(P>0.05)$. The quality of life improvement in the CRF group was maintained for a long time. At 6 and 12 months after treatment, the ODI was lower than that in the PRF group, and the difference was significant $(P<0.05)$ (Table 3$)$.

\section{SF-36 before and after treatment}

After the relief of pain, both groups received different degrees of improvement in the quality of life, including physical function, physical role, bodily pain, general health, vitality, social function, emotional role, and mental health. At each observation time point, PCS and MCS in both groups were

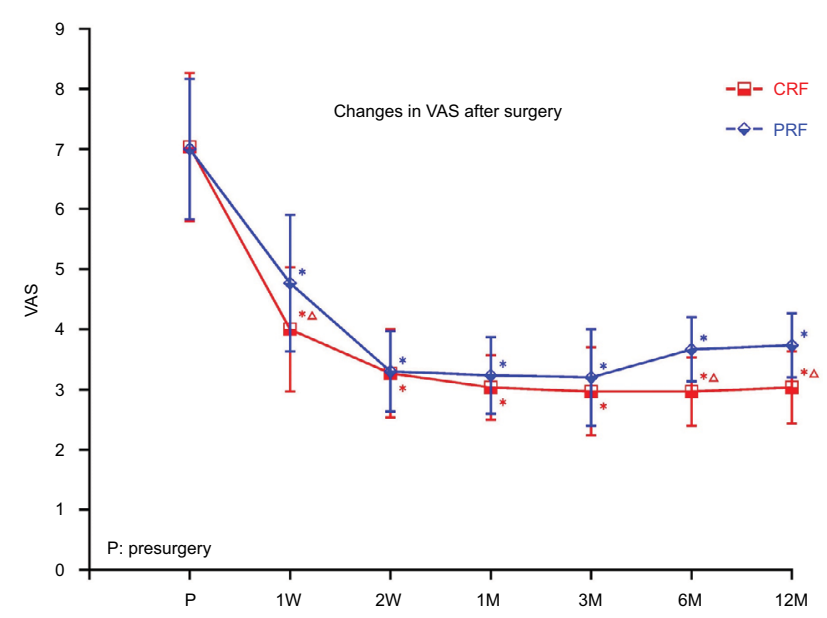

Figure 3 The comparison of VAS in two groups (mean $\pm S D$ ).

Notes: Data are presented as mean \pm SD. Compared to pretreatment value, ${ }^{*} P<0.05$ CRF group compared with the PRF group, $\triangle P<0.05$.

Abbreviations: CRF, conventional radiofrequency; $M$, months; PRF, pulsed radiofrequency; $\mathrm{W}$, weeks. increased after treatment. Compared with the pretreatment value, the difference was significant $(P<0.05)$; PCS and MCS in both groups increased gradually. The increase was most obvious at 3 months and decreased at 6 and 12 months. The CRF group improved quickly, and PCS and MCS increased significantly at 1 week after treatment. Compared to the PRF group, the difference was significant $(P<0.05)$. The improvement of the PRF group was relatively slow. At 2 weeks, and 1 and 3 months, there was no significant difference between the two groups $(P>0.05)$. In the CRF group, the improvement of the quality of life was maintained for a long time. At 6 and 12 months after treatment, PCS and MCS were higher than those in the PRF group, and the difference was significant $(P<0.05)$ (Figure 4).

\section{Discussion}

SIJP is difficult to distinguish from other sources of low back pain through medical history and physical examination. The innervation is complicated, so the problem of SIJ is easily overlooked. ${ }^{1,13}$ The incidence of SIJP caused by various reasons is increasing, and the treatment of SIJP poses a huge challenge and requires extensive attention.

The SIJ has a deep position and special shape, with unique characteristics. ${ }^{2}$ Early sacroiliac arthritis occurs in the synovial membrane, and the iliac side is more important than the sacrum side. ${ }^{14}$ Therefore, the puncture target should choose the synovial part, and the needle should also face the iliac side. Anatomically, the individual differences in distance between the posterior edge and the midline and the angle of the SIJ are large. Therefore, accurate positioning is very difficult. The X-ray fluoroscopy cannot guide the puncture needle into the SIJ due to its overlapping structure and lowdensity resolution; while the density resolution and the spatial

Table 2 Comparison of the total efficiency rates after treatment in two groups

\begin{tabular}{|l|l|l|l|l|l|l|}
\hline & $\mathbf{n}$ & CR & PR & MR & NR & Total efficiency (\%) \\
\hline CRF & 32 & $6(18.8 \%)$ & $7(21.9 \%)$ & $5(15.6 \%)$ & $14(43.7 \%)$ & $18(56.3 \%)$ \\
PRF & 32 & $3(9.4 \%)$ & $5(15.6 \%)$ & $2(6.3 \%)$ & $22(68.7 \%)$ & $10(31.3 \%)$ \\
\hline
\end{tabular}

Note: Compared to the PRF group, ${ }^{*} P<0.05$.

Abbreviations: CR, complete remission; CRF, conventional radiofrequency; MR, mild remission; NR, no remission; PR, partial remission; PRF, pulsed radiofrequency.

Table 3 Comparison of ODI in two groups

\begin{tabular}{|l|l|l|l|l|l|l|}
\hline Group & Before & \multicolumn{4}{l|}{ After treatment } \\
\cline { 3 - 7 } & treatment & I week & 2 weeks & I month & 3 months & 6 months \\
\hline CRF & $48.67 \pm I I .02$ & $35.94 \pm 7.64^{*} \triangle$ & $32.52 \pm 6.23^{*}$ & $32.14 \pm 6.10^{*}$ & $28.76 \pm 5.78^{*}$ & $28.93 \pm 4.78^{*} \triangle$ \\
PRF & $48.92 \pm 10.42$ & $41.36 \pm 8.5 I^{*}$ & $32.91 \pm 6.47^{*}$ & $32.25 \pm 5.97^{*}$ & $30.84 \pm 6.23^{*}$ & $33.67 \pm 5.54^{*}$ \\
\hline
\end{tabular}

Notes: Data are presented as mean \pm SD (\%). Compared to the pretreatment value, ${ }^{*} P<0.05$; CRF group compared to the $P R F$ group, $\triangle P<0.05$.

Abbreviations: CRF, conventional radiofrequency; ODI, Oswestry disability index; PRF, pulsed radiofrequency. 

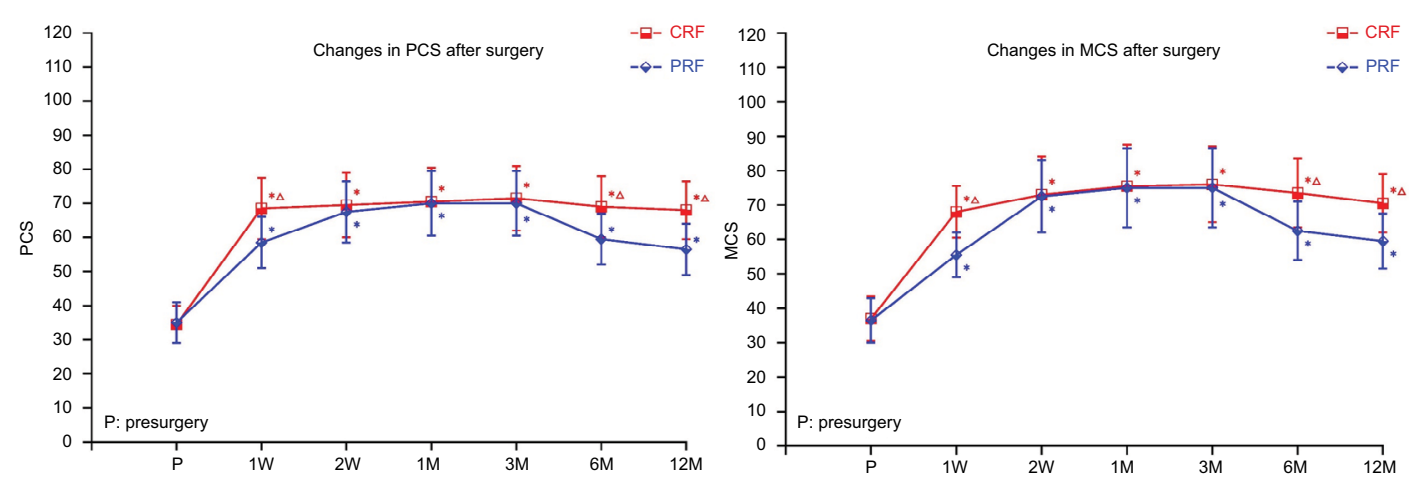

Figure 4 The comparison of 36-item short-form health survey before and after treatment in two groups.

Notes: Results are presented as mean \pm SEM. Compared to the pretreatment value, ${ }^{*} P<0.05 ;$ CRF group compared to the PRF group, $\triangle P<0.05$.

Abbreviations: CRF, conventional radiofrequency; MCS, mental component summary; PCS, physical component summary; PRF, pulsed radiofrequency.

resolution in the plane of the $\mathrm{CT}$ are high, there is no overlapping interference. It is an effective positioning and guiding method for the SIJ synovia puncture. Positioning under CT guidance, the angle and depth can be clearly defined, and the SIJ synovia can be directly penetrated through the ligament. In this study, all patients completed the treatment successfully. In the CT scan image, it was observed that RF needle tip was located at the junction of the middle $1 / 3$ and the lower $1 / 3$ of the SIJ, where all or most of the SIJ was the synovial membrane ${ }^{15}$ and the direction was relatively straight and suitable for puncture; three-dimensional CT could further clarify the position and showed that the RF needle was located at the SIJ. During the puncture, it was necessary to avoid puncturing the pelvic cavity and damaging the pelvic structure (the posterior wall of the pelvis, the internal iliac vessel, and the lumbosacral trunk near the lower $1 / 3$ of the SIJ). The puncture RF needle was tilted slightly to the iliac side to reduce the chance of penetrating into the pelvic cavity. Before the radiofrequency treatment, the sensory and exercise tests were further performed. No hips and the lower extremity muscles were induced to tremble and no pain was induced, then the radiofrequency treatment was performed. After treatment, patients experienced minor complications such as local swelling, pain at the needle puncture site, dizziness, flushing, sweating, nausea, and hypotension, but those were short-lived and self-limiting. In this study, no serious complications such as spinal cord injury and limb weakness occurred.

At present, there are various treatment options for sacroiliac pain, including conservative treatment, interventional therapy, and surgical treatment. The treatment is selected according to the patient's condition, and conservative treatment is preferred. Most patients may have a better curative effect. However, some patients have poor conservative treatment effect and the course of the disease is prolonged. At this time, interventional therapy can be further considered. Interventional therapy has the advantages of small trauma, quick recovery, short operation time, and short postoperative hospital stay, while the surgical treatment is often the last choice because of the large trauma. IA injection of local anesthetics and cortisol directly into the joints can quickly decrease inflammation in and around the joints, relieve pain, and promote tissue repair, but the maintenance time is short and repeated treatment always has more side effects. ${ }^{5}$ RFD is one of the most rapidly developing technology in recent years. In some intractable sacroiliac pain, some scholars had achieved good results by using radiofrequency thermocoagulation. Studies had shown that RFD was effective in treating chronic refractory SIJP. The effective rate of 6 months after treatment could reach $49.9 \%,{ }^{16}$ and in some studies, the effective rate of 9 months after RFD reached $89 \%{ }^{17}$ and could provide significant relief for up to one year. ${ }^{3}$ Gevargez et $\mathrm{al}^{18}$ applied CRF to the posterior interosseous sacroiliac ligaments and the dorsal rami of the L5 spinal nerve. No pain was reported by $34.2 \%$, and significant pain relief was experienced by $31.6 \%$. Cohen et a $\mathrm{a}^{19}$ retrospectively evaluated 40 patients in two institutions. The patients received RFD of L4-L5 primary dorsal rami and S1-S3 lateral branches. Fifty-two percent obtained positive results and believed that the CRF was effective in the treatment of sacroiliac pain, and the long-term effect was satisfactory. ${ }^{3}$ However, van Tilburg $\mathrm{CW}$ et $\mathrm{a}^{20}$ performed radiofrequency on the S1-S4 nerve root lateral branches and L5 posterior branch. Compared with the sham group, the hypothesis of no difference in the pain reduction or global perceptual effect cannot be rejected. CRF could cause nerve damage, which easily led to local sensory 
retardation, sensory degeneration, abnormal pain, persistent weakness in the lower limbs, and other complications such as burning and numbness. As the maximum temperature of the PRF electrode does not exceed $42^{\circ} \mathrm{C}$, there is no significant nerve damage in the PRF compared to the CRF. Ahadian ${ }^{21}$ found that PRF was similar to CRF on demonstrating response rates for facet arthropathy, sacroiliac arthropathy, and many other chronic pain conditions. Vallejo et $\mathrm{a}^{22}$ performed pulsed RFD on the lateral branch of the medial branch of L4, posterior primary rami of L5, and lateral branches S1-S2. A "good" (VAS decrease $>50 \%$ ) or "excellent" (VAS decrease $>80 \%$ ) pain relief was experienced by $72.7 \%$, the long-term effect was satisfactory, and it was effective for the treatment of intractable SIJ dysfunction. At present, there are many studies on radiofrequency treatment of local ligament and denervation, but few studies on IA SIJ radiofrequency. Schianchi et al performed IA PRF on the shoulder, knee, trapezio-metacarpal, and first metatarsophalangeal joints, and the pain in most of the joints was significantly alleviated. ${ }^{23}$ Studies had shown that PRF was superior to CRF in small joint-derived pain. ${ }^{24,25}$ The use of IA PRF in advanced knee osteoarthritis was also effective and safe, ${ }^{26}$ but the efficacy on the large joint of the SIJ was still unclear.

In this study, IA CRF and PRF were used to treat sacroiliac pain. It was found that in both groups, the VAS and ODI decreased, and PCS and MCS increased. Compared to the pretreatment value, the difference was significant in both groups $(P<0.05)$, indicating that both treatments were effective and could alleviate the symptoms of SIJP. The analgesia effect in the CRF group was rapid and the quality of life improved in the early stage. The VAS decreased significantly and the quality of life improved 1 week after treatment. The pain relief and quality-of-life improvement could sustain for a long time, up to 6 months and 12 months after treatment, and the difference was significant between the two groups $(P<0.05)$. In the PRF group, the effect was slow, and VAS and ODI decreased gradually. The same analgesic effect as CRF was achieved at 2 weeks, and 1 and 3 months, but the maintenance time was short. CRF is a neurodestructive technique. The high-frequency current causes the ions in the tissue to oscillate, causing local heating. The $\mathrm{A} \delta$ and $\mathrm{C}$-type nerve fiber that transmits pain are coagulated and denatured by heating. As the conductive tactile nerve fibers $(A \alpha$ and $A \beta)$ can tolerate relatively high temperatures, they are not damaged. CRF blocks the action potential by cutting off the sensory pathway and then the pain relieves. ${ }^{27,28}$ While PRF does not damage the nerve, pain relief may be related to reversible neurons temporar- ily blocking nerve signals through the nerve conduction pathway. PRF could inhibit MAPK activation, reduced cytokine release, and inhibited the excitatory amino acids release in the spinal cord. ${ }^{29}$ Moreover, PRF could attenuate JNK activation in spinal dorsal horn, ${ }^{30}$ inhibited spinal cord sensitization, and regulated the expression of multiple genes in the pathway. The expressions of anti-inflammatory factor genes (GABAB-R1, Na/K-ATPase, and 5-HT3r) were enhanced, while the expressions of proinflammatory factor genes (TNF- $\alpha$ and IL-6) were decreased, ${ }^{31}$ so the pain was relieved. ${ }^{32}$ Therefore, the analgesic effect of PRF was slow and its long-term analgesic effect might be related to neuromodulation. The total efficiency rate in the CRF and PRF groups was $56.3 \%$ and $31.3 \%$ at 12 months after treatment. The difference between the two groups was significant $(P<0.05)$. The action point of this study was located in IA SIJ, which caused no nerve damage and can be safely treated with CRF. The IA SIJ radiofrequency is different from RFD. Nerves are segmental distribution requiring multiple segments for ablation, while the volume of the SIJ is very limited, and the surrounding bone also has an insulating property. In the joint, part of the current is deflected by the bone surface, retaining the current intensity and electric field in the joint space and not being diluted rapidly at long distances. Therefore, the current intensity and electric field in IA SIJ will be higher than that of the electrode in the soft tissue, so it has a superior analgesic effect. ${ }^{33}$ Thus, we chose only one location for radiofrequency ablation. However, the position of the RF needle was required to be accurate, that was why we chose CT-guided one.

This study had several limitations. The study was a retrospective study, and it was already known that the efficacy of simple SIJ block injections was short, so we did not set up a control group for the patient's treatment effect. Patients with postoperative SIJ pain were not included in our inclusion criteria, so the indications for the use of radiofrequency were relatively inadequate. At the same time, mechanisms of the RF should be further studied, such as cytokines.

In summary, CT-guided IA PRF and CRF in the treatment of sacroiliac pain is safe and effective. It could significantly alleviate sacroiliac pain, reduce ODI, and improve the quality of life physically and mentally. CRF is superior to PRF in the early and late stages. It is recommended for the treatment of SIJP.

\section{Acknowledgment}

This study was supported by the Natural Science Foundation of Liaoning Province (No. 20170541032). 


\section{Disclosure}

The authors report no conflicts of interest in this work.

\section{References}

1. Vanelderen P, Szadek K, Cohen SP, et al. 13. Sacroiliac joint pain. Pain Pract. 2010;10(5):470-478.

2. Forst SL, Wheeler MT, Fortin JD, Vilensky JA. The sacroiliac joint: anatomy, physiology and clinical significance. Pain Physician. 2006;9(1):61-67.

3. Cohen SP, Chen Y, Neufeld NJ. Sacroiliac joint pain: a comprehensive review of epidemiology, diagnosis and treatment. Expert Rev Neurother. 2013;13(1):99-116.

4. Dreyfuss P, Dreyer SJ, Cole A, Mayo K. Sacroiliac joint pain. JAm Acad Orthop Surg. 2004;12(4):255-265.

5. D'Orazio F, Gregori LM, Gallucci M. Spine epidural and sacroiliac joints injections--when and how to perform. Eur J Radiol. 2015;84(5):777-782.

6. Chua NH, Vissers KC, Sluijter ME. Pulsed radiofrequency treatment in interventional pain management: mechanisms and potential indicationsa review. Acta Neurochir (Wien). 2011;153(4):763-771

7. Bhatia A, Peng P, Cohen SP. Radiofrequency procedures to relieve chronic knee pain: an evidence-based narrative review. Reg Anesth Pain Med. 2016;41(4):501-510.

8. Elawamy A, Abdalla EEM, Shehata GA. Effects of pulsed versus conventional versus combined radiofrequency for the treatment of trigeminal neuralgia: a prospective study. Pain Physician. 2017;20(6):E873-E881.

9. Choi S, Choi HJ, Cheong Y, Chung SH, Park HK, Lim YJ. Inflammatory responses and morphological changes of radiofrequency-induced rat sciatic nerve fibres. Eur J Pain. 2014;18(2):192-203.

10. Lee JB, Byun JH, Choi IS, Kim Y, Lee JS. The effect of pulsed radiofrequency applied to the peripheral nerve in chronic constriction injury rat model. Ann Rehabil Med. 2015;39(5):667-675.

11. Merskey H, Bogduk N. Classification of Chronic Pain: Descriptions of Chronic Pain Syndromes and Definitions of Pain Terms. 2nd ed. Seattle, WA: IASP Press; 1994.

12. Lam CL, Tse EY, Gandek B, Fong DY. The SF-36 summary scales were valid, reliable, and equivalent in a Chinese population. JClin Epidemiol. 2005;58(8):815-822.

13. Slipman CW, Whyte WS 2nd, Chow DW, Chou L, Lenrow D, Ellen M. Sacroiliac joint syndrome. Pain Physician. 2001;4(2):143-152.

14. Puhakka KB, Melsen F, Jurik AG, Boel LW, Vesterby A, Egund N. MR imaging of the normal sacroiliac joint with correlation to histology. Skeletal Radiol. 2004;33(1):15-28.

15. Foley BS, Buschbacher RM. Sacroiliac joint pain: anatomy, biomechanics, diagnosis, and treatment. Am J Phys Med Rehabil. 2006;85(12):997-1006.

16. Aydin SM, Gharibo CG, Mehnert M, Stitik TP. The role of radiofrequency ablation for sacroiliac joint pain: a meta-analysis. $P M R$. 2010;2(9):842-851.

17. Cohen SP, Abdi S. Lateral branch blocks as a treatment for sacroiliac joint pain: a pilot study. Reg Anesth Pain Med. 2003;28(2):113-119.
18. Gevargez A, Groenemeyer D, Schirp S, Braun M. CT-guided percutaneous radiofrequency denervation of the sacroiliac joint. Eur Radiol. 2002;12(6):1360-1365.

19. Cohen SP, Strassels SA, Kurihara C, et al. Outcome predictors for sacroiliac joint (lateral branch) radiofrequency denervation. Reg Anesth Pain Med. 2009;34(3):206-214.

20. van Tilburg CW, Schuurmans FA, Stronks DL, Groeneweg JG, Huygen FJ. Randomized sham-controlled double-blind multicenter clinical trial to ascertain the effect of percutaneous radiofrequency treatment for sacroiliac joint pain: three-month results. Clin J Pain. 2016;32(11):921-926.

21. Ahadian FM. Pulsed radiofrequency neurotomy: advances in pain medicine. Curr Pain Headache Rep. 2004;8(1):34-40.

22. Vallejo R, Benyamin RM, Kramer J, Stanton G, Joseph NJ. Pulsed radiofrequency denervation for the treatment of sacroiliac joint syndrome. Pain Med. 2006;7(5):429-434.

23. Schianchi PM, Sluijter ME, Balogh SE. The treatment of joint pain with intra-articular pulsed radiofrequency. Anesth Pain Med. 2013;3(2):250-255.

24. Kroll HR, Kim D, Danic MJ, Sankey SS, Gariwala M, Brown M. A randomized, double-blind, prospective study comparing the efficacy of continuous versus pulsed radiofrequency in the treatment of lumbar facet syndrome. J Clin Anesth. 2008;20(7):534-537.

25. Tekin I, Mirzai H, Ok G, Erbuyun K, Vatansever D. A comparison of conventional and pulsed radiofrequency denervation in the treatment of chronic facet joint pain. Clin J Pain. 2007;23(6):524-529.

26. Eyigor C, Eyigor S, Akdeniz S, Uyar M. Effects of intra-articular application of pulsed radiofrequency on pain, functioning and quality of life in patients with advanced knee osteoarthritis. J Back Musculoskelet Rehabil. 2015;28(1):129-134.

27. Zhou X, Liu Y, Yue Z, Luan D, Zhang H, Han J. Comparison of nerve combing and percutaneous radiofrequency thermocoagulation in the treatment for idiopathic trigeminal neuralgia. Braz J Otorhinolaryngol. 2016;82(5):574-579.

28. Letcher FS, Goldring S. The effect of radiofrequency current and heat on peripheral nerve action potential in the cat. $J$ Neurosurg. 1968;29(1):42-47.

29. Yang $\mathrm{CH}$, Chen KH, Huang HW, Sheen-Chen SM, Lin CR. Pulsed radiofrequency treatment attenuates increases in spinal excitatory amino acid release in rats with adjuvant-induced mechanical allodynia. Neuroreport. 2013;24(8):431-436.

30. Chen KH, Yang CH, Juang SE, et al. Pulsed radiofrequency reduced complete Freund's adjuvant-induced mechanical hyperalgesia via the spinal c-Jun N-terminal kinase pathway. Cell Mol Neurobiol. 2014;34(2):195-203.

31. Vallejo R, Tilley DM, Williams J, Labak S, Aliaga L, Benyamin RM. Pulsed radiofrequency modulates pain regulatory gene expression along the nociceptive pathway. Pain Physician. 2013;16(5):E601-E613.

32. Lin ML, Lin WT, Huang RY, et al. Pulsed radiofrequency inhibited activation of spinal mitogen-activated protein kinases and ameliorated early neuropathic pain in rats. Eur J Pain. 2014;18(5):659-670.

33. Sluijter ME, Teixeira A, Serra V, Balogh S, Schianchi P. Intra-articular application of pulsed radiofrequency for arthrogenic pain - report of six cases. Pain Pract. 2008;8(1):57-61.
Journal of Pain Research

\section{Publish your work in this journal}

The Journal of Pain Research is an international, peer reviewed, open access, online journal that welcomes laboratory and clinical findings in the fields of pain research and the prevention and management of pain. Original research, reviews, symposium reports, hypothesis formation and commentaries are all considered for publication.
Dovepress

The manuscript management system is completely online and includes a very quick and fair peer-review system, which is all easy to use. Visit http://www.dovepress.com/testimonials.php to read real quotes from published authors. 